\title{
Quasi-Optimal Estimates for Finite Element Approximations Using Orlicz Norms*
}

\author{
By Ricardo G. Durán
}

\begin{abstract}
We consider the approximation by linear finite elements of the solution of the Dirichlet problem $-\Delta u=f$. We obtain a relation between the error in the infinite norm and the error in some Orlicz spaces. As a consequence, we get quasi-optimal uniform estimates when $u$ has second derivatives in the Orlicz space associated with the exponential function. This estimate contains, in particular, the case where $f$ belongs to $L^{\infty}$ and the boundary of the domain is regular. We also show that optimal order estimates are valid for the error in this Orlicz space provided that $u$ be regular enough.
\end{abstract}

1. Introduction. Consider the problem of finding $u$ such that

$$
\left\{\begin{aligned}
-\Delta u=f & \text { in } \Omega, \\
u=0 & \text { on } \partial \Omega,
\end{aligned}\right.
$$

where $\Omega$ is a bounded domain contained in $R^{n}$ and $f$ is a given function.

We shall use standard notation for the Sobolev spaces $W_{p}^{k}(\Omega)$ and $H^{k}(\Omega)=W_{2}^{k}$ with the norms

$$
\|f\|_{k, p, \Omega}=\sum_{j \leqslant k}|f|_{j, p, \Omega}
$$

where

$$
|f|_{j, p, \Omega}=\sum_{|\alpha|=j}\left\|D^{\alpha} f\right\|_{L^{p}(\Omega)}
$$

We shall write $\|f\|_{k, p}=\|f\|_{k, p, \Omega}$ and $|f|_{k, p}=|f|_{k, p, \Omega}$ when there is no confusion.

The letter $C$ will denote a constant, not necessarily the same at each occurrence.

For simplicity we will consider $\Omega$ to be a convex polyhedral domain, but the results are valid in more general domains as in [9].

Let $\left\{\mathscr{T}_{h}\right\}$ be a quasi-regular family of triangulations of $\Omega$ and denote by $u_{h}$ the $H_{0}^{1}$-projection of $u$ into the space of piecewise linear functions $M_{h} \subset H_{0}^{1}$, that is,

$$
\int_{\Omega} \nabla u_{h} \nabla v_{h} d x=\int_{\Omega} f v d x, \quad v_{h} \in M_{h}
$$

It is well known (see [1]) that

$$
\left|u-u_{h}\right|_{0,2} \leqslant C h^{2}|u|_{2,2} \text { and }\left|u-u_{h}\right|_{1,2} \leqslant C h|u|_{2,2} \text {. }
$$

Received September 16, 1985; revised May 15, 1986.

1980 Mathematics Subject Classification (1985 Revision). Primary 65N30.

* Supported by Consejo Nacional de Investigaciones Científicas y Técnicas de Argentina. 
Many authors have studied estimates for $u-u_{h}$ in $W_{p}^{1}$-norms and $L^{p}$-norms. In [8] the following optimal estimate for the gradient of the error in $L^{p}$ is obtained,

$$
\left|u-u_{h}\right|_{1, p} \leqslant C h\|u\|_{2, p} \text { for } 1<p \leqslant \infty .
$$

Then by the usual duality argument (see [1]) they get

$$
\left|u-u_{h}\right|_{0, p} \leqslant C h^{2}\|u\|_{2, p} \text { for } 2 \leqslant p<\infty,
$$

provided that $\Omega$ is a convex polygonal domain or $\partial \Omega$ is smooth.

As is known, this duality argument cannot be applied for $p=\infty$.

A quasi-optimal estimate for the error in $L^{\infty}$ was obtained in [9], where it is proved that

$$
\left|u-u_{h}\right|_{0, \infty} \leqslant C h^{2} \log \frac{1}{h}\|u\|_{2, \infty} .
$$

Moreover, in [4] an example is given that shows that the logarithm in this estimate cannot be removed.

We will work here with Orlicz spaces defined in the following way. Given a convex function $\phi: R_{+} \rightarrow R_{+}, \phi(0)=0$, let

$$
L^{\phi}(\Omega)=\left\{f|\exists b>0| \int_{\Omega} \phi\left(\frac{|f|}{b}\right) d x<\infty\right\} .
$$

$L^{\phi}$ is a Banach space with the norm

$$
\|f\|_{L^{\phi}}=\inf \left\{b>0 \mid \int_{\Omega} \phi\left(\frac{|f(x)|}{b}\right) d x \leqslant 1\right\} .
$$

We will call $W_{\phi}^{k}$ the space of functions in $L^{\phi}$ with derivatives up to the order $k$ in $L^{\phi}$, and we will use analogous notation as in the $L^{p}$ case for the norms and seminorms.

When the boundary of $\Omega$ is regular and $1<p<\infty$ [3],

$$
\|u\|_{2, p} \leqslant C|f|_{0, p}
$$

and consequently,

$$
\left|u-u_{h}\right|_{0, p} \leqslant C h^{2}|f|_{0, p} .
$$

As is well known, the regularity result mentioned above is not true for $p=\infty$, but if $f \in L^{\infty}$ the solution $u \in W_{\phi_{1}}^{2}$, where $\phi_{1}(t)=e^{t}-t-1$. Moreover, the second derivatives of $u$ are in the space of functions with bounded mean oscillation BMO (same proof as in the $L^{p}$ case [3], using the result of [6]) and this space is contained in $L^{\phi_{1}}$ when the domain is bounded, [5]. Then it is natural to seek an estimate for $\left|u-u_{h}\right|_{0, \infty}$ when $u$ has second derivatives in $L^{\phi_{1}}$.

In this paper we obtain a relation between the error in $L^{\infty}$ and the error in some Orlicz spaces that implies in particular the following quasi-optimal estimate,

$$
\left|u-u_{h}\right|_{0, \infty}<C h^{2}\left(\log \frac{1}{h}\right)^{2}\|u\|_{2, \phi_{1}} .
$$

This estimate contains as a particular case the following one proved in [9],

$$
\left|u-u_{h}\right|_{0, \infty}<C h^{2}\left(\log \frac{1}{h}\right)^{2}|f|_{0, \infty}
$$


A similar estimate was obtained also in [7] but with a higher power of the logarithm and with the BMO norm of the second derivatives in the right-hand side.

Our result is more general because BMO is strictly contained in $L^{\phi_{1}}$ (for example, in $\Omega=(-1,1)$ the function

$$
f(x)= \begin{cases}\log x, & x>0 \\ 0, & x<0\end{cases}
$$

is in $L^{\phi_{1}}$ but not in BMO).

Error estimates for problems where $u$ has other kinds of singularities can be obtained by our theorem. As examples, consider $\Omega=\left\{x \in R^{2}|| x \mid<1 / e\right\}$ and

$$
u(x)=|x|^{2}\left(\log \frac{1}{|x|}\right)^{1 / n}-1 / e^{2}, \quad n \in N .
$$

In this case, $D^{\alpha} u \in L^{\phi}(\Omega)$ for $|\alpha|=2$, where $\phi(t)=e^{t^{n}}-t^{n}-1$, and then we will get the following estimate,

$$
\left|u-u_{h}\right|_{0, \infty} \leqslant C h^{2}\left(\log \frac{1}{h}\right)^{1+1 / n}\|u\|_{2, \phi}
$$

Finally, we show in the two-dimensional case that

$$
\left|u-u_{h}\right|_{0, \phi_{1}} \leqslant C h^{2}\|u\|_{2, \infty},
$$

provided that $\partial \Omega$ is smooth or $\Omega$ is a Lipschitz convex domain. In this way we show that the logarithm factor can be removed if we replace the $L^{\infty}$-norm on the left by a slightly weaker Orlicz norm.

\section{Error Estimates.}

LEMMA 1. If $v \in M_{h}$ the following inverse inequality holds,

$$
|v|_{0, \infty} \leqslant C \phi^{-1}\left(1 / h^{n}\right)|v|_{0, \phi} .
$$

Proof. Let $T \in \mathscr{T}_{h}$ such that $|v|_{0, \infty, T}=|v|_{0, \infty}$. By usual scaling arguments one can see that

$$
|v|_{0, \infty, T} \leqslant C\left(1 / h^{n}\right) \int_{T}|v(x)| d x
$$

Let $\psi$ be the complementary function of $\phi$; then we can apply the Hölder inequality for Orlicz spaces, and we have

$$
|v|_{0, \infty, T} \leqslant C\left(1 / h^{n}\right)|v|_{0, \phi}|\chi|_{0, \psi},
$$

where $\chi$ is the characteristic function of $T$. But $|\chi|_{0, \psi}=b$, where $b$ satisfies

$$
\int_{T} \psi(1 / b) d x=1
$$

so $b=1 / \psi^{-1}(1 /|T|)$ and then, using the inequality $t \leqslant \phi^{-1}(t) \psi^{-1}(t)$, we get

$$
b \leqslant|T| \phi^{-1}(1 /|T|) \leqslant C h^{n} \phi^{-1}\left(1 / h^{n}\right),
$$

and (2) and (3) imply (1).

The result of the following lemma is proved in [2] but we give here a more direct proof. 
LEMMA 2. Let $g$ be a continuous function such that $\partial g / \partial x_{j} \in L^{\phi}(Q)$, where $Q \subset R^{n}$ is an open set with Lipschitz boundary. Assume that

$$
\mu(t)=\int_{0}^{t} \phi^{-1}\left(1 / s^{n}\right) d s
$$

is finite. Then,

$$
|g(x+y)-g(x)| \leqslant C|g|_{1, \phi, Q} \mu(|y|) .
$$

Proof. Taking an extension, we can assume that $g$ is in $W_{\phi}^{1}\left(R^{n}\right)$. Let $\eta \in C_{0}^{\infty}$ such that $\int \eta=1$ and $0 \leqslant \eta(x) \leqslant 1, \eta_{t}(x)=t^{-n} \eta(x / t)$ and $v(x, t)=g * \eta_{t}(x)$; then

$$
\left(\partial v / \partial x_{j}\right)(x, t)=\int\left(\partial g / \partial x_{j}\right)(y) \eta_{t}(x-y) d y,
$$

and applying the Hölder inequality, we have

$$
\left|\left(\partial v / \partial x_{j}\right)(x, t)\right| \leqslant 2\left|\partial g / \partial x_{j}\right|_{0, \phi}\left|\eta_{t}\right|_{0, \psi}
$$

Set $b=t^{-n} / \psi^{-1}\left(t^{-n}\right)$; then, since $\eta(x / t) \leqslant 1$ and $\psi$ is convex, we have

$$
\int \psi\left(t^{-n} \eta(x / t) / b\right) d x=\int \psi\left(\psi^{-1}\left(t^{-n}\right) \eta(x / t)\right) d x \leqslant \int \eta(x / t) t^{-n} d x=1 .
$$

Consequently,

$$
\left|\eta_{t}\right|_{0, \psi} \leqslant t^{-n} / \psi^{-1}\left(t^{-n}\right) \leqslant \phi^{-1}\left(t^{-n}\right)
$$

and by (5),

$$
\left|\left(\partial v / \partial x_{j}\right)(x, t)\right| \leqslant 2\left|\partial g / \partial x_{j}\right|_{0, \phi} \phi^{-1}\left(t^{-n}\right) .
$$

A similar estimate for $\partial v / \partial t$ can be obtained in the following way. First observe that

$$
\partial \eta_{t} / \partial t=-\sum_{i=1}^{n} \partial\left(x_{i} \eta\right)_{t} / \partial x_{i}
$$

then,

$$
\begin{aligned}
(\partial v / \partial t)(x, t) & =\left(g * \partial \eta_{t} / \partial t\right)(x)=-\sum_{i=1}^{n}\left(g * \partial\left(x_{i} \eta\right)_{t} / \partial x_{i}\right) \\
& =-\sum_{i=1}^{n} \partial g / \partial x_{i} *\left(x_{i} \eta\right)_{t},
\end{aligned}
$$

and now we are in the same situation as before, with $\eta$ replaced by $x_{i} \eta$. In the same way we can prove that

$$
\left|\left(x_{i} \eta\right)_{t}\right|_{0, \psi} \leqslant \phi^{-1}\left(t^{-n}\right) \max \left\{\left\|x_{i} \eta\right\|_{L^{1}},\left\|x_{i} \eta\right\|_{L^{\infty}}\right\}
$$

and then,

$$
|(\partial v / \partial t)(x, t)| \leqslant C|g|_{1, \phi} \phi^{-1}\left(t^{-n}\right),
$$

where $C$ depends on $\eta$. 
Now (4) follows easily, writing

$$
\begin{aligned}
g(x+y)-g(x)= & {[g(x+y)-v(x+y,|y|)]+[v(x+y,|y|)-v(x,|y|)] } \\
& +[v(x,|y|)-g(x)]
\end{aligned}
$$

and estimating each summand separately.

Now we restrict ourselves to functions of the form $\phi(t)=\sum_{j=2}^{\infty} a_{j} t^{j}$ with $a_{j} \geqslant 0$, because our main example is of this form. For this class of functions it is easy to prove results about the error for Lagrange interpolation in the $\phi$-norm. In fact, using the known estimates for $L^{p}$-norms and the series expansion of $\phi$, we get the following result,

$$
\left|u-I_{h} u\right|_{j, \phi} \leqslant C h^{2-j}\|u\|_{2, \phi}, \quad j=0,1,
$$

where $I_{h} u$ is the Lagrange interpolation of $u$. Then we can state the following corollary of Lemma 2.

COROllary 1. Let $\phi(t)=\sum_{j=2}^{\infty} a_{j} t^{j}, a_{j} \geqslant 0$, be an Orlicz function; then

$$
\left|u-I_{h} u\right|_{0, \infty} \leqslant C h \mu(h)\|u\|_{2, \phi} .
$$

We can now give a theorem which compares the error in $L^{\infty}$ - and $L^{\phi}$-norms.

THEOREM 1. If $\phi$ satisfies the condition of Corollary 1 and $\mu$ is the function associated with $\phi$ in Lemma 2 , then there exists a constant $C$ such that

$$
\left|u-u_{h}\right|_{0, \infty} \leqslant C h \mu(h)\left[\|u\|_{2, \phi}+\frac{\left|u-u_{h}\right|_{0, \phi}}{h^{2}}\right] .
$$

Proof. By Lemma 1 and Corollary 1 we have

$$
\begin{aligned}
\left|u-u_{h}\right|_{0, \infty} & \leqslant\left|u-I_{h} u\right|_{0, \infty}+\left|I_{h} u-u_{h}\right|_{0, \infty} \\
& \leqslant C\left[h \mu(h)\|u\|_{2, \phi}+\phi^{-1}\left(1 / h^{n}\right)\left|I_{h} u-u_{h}\right|_{0, \phi}\right] .
\end{aligned}
$$

But $\left|I_{h} u-u\right|_{0, \phi} \leqslant C h^{2}\|u\|_{2, \phi}$ and then,

$$
\left|u-u_{h}\right|_{0, \infty} \leqslant C\left[h \mu(h)\|u\|_{2, \phi}+h^{2} \phi^{-1}\left(h^{-n}\right)\|u\|_{2, \phi}+\phi^{-1}\left(h^{-n}\right)\left|u-u_{h}\right|_{0, \phi}\right] .
$$

Noting that $h \phi^{-1}\left(h^{-n}\right) \leqslant \mu(h)$, we obtain the result.

COROLlaRy 2. There exists a constant $C$ such that

$$
\left|u-u_{h}\right|_{0, \infty} \leqslant C h\left(\log h^{-1}\right) \mu(h)\|u\|_{2, \phi}
$$

and, in particular,

$$
\left|u-u_{h}\right|_{0, \infty}<C h^{2}\left(\log h^{-1}\right)^{2}\|u\|_{2, \phi_{1}} \text {. }
$$

Proof. By the known estimates [9], [1]

$$
\left|u-u_{h}\right|_{0, \infty} \leqslant C h^{2} \log h^{-1}\|u\|_{2, \infty} \quad \text { and } \quad\left|u-u_{h}\right|_{0,2} \leqslant C h^{2}\|u\|_{2,2}
$$

we get by interpolation

$$
\left|u-u_{h}\right|_{0, p} \leqslant C h^{2} \log h^{-1}\|u\|_{2, p} \text { for } 2 \leqslant p<\infty,
$$

with $C$ independent of $p$. Using the expansion in power series of $\phi$, we get

$$
\left|u-u_{h}\right|_{0, \phi}<C h^{2} \log h^{-1}\|u\|_{2, \phi},
$$

hence, by Theorem 1, we get (6). 
When $\phi=\phi_{1}$ it is easily shown that $\mu_{1}(h) \leqslant C h \log h^{-1}$ for small $h$ and this proves (7).

We will show in the following theorem that as a consequence of the estimates for $\left|u-u_{h}\right|_{1, \infty}$ [8] we have optimal-order estimates in the $\phi_{1}$-norm if $u \in W_{\infty}^{2}$.

THEOREM 2. Let $\Omega \subset R^{2}$ be such that $\partial \Omega$ is smooth or $\Omega$ is convex with Lipschitz boundary. Then there exists a constant $C$ such that

$$
\left|u-u_{h}\right|_{0, \phi_{1}} \leqslant C h^{2}\|u\|_{2, \infty} \text {. }
$$

Proof. In [8] it is proved that

$$
\left|u-u_{h}\right|_{1, p} \leqslant C h\|u\|_{2, p}, \quad 2 \leqslant p \leqslant \infty .
$$

On the other hand, if $v \in H_{0}^{1}(\Omega)$ and $-\Delta v=g$,

$$
\|v\|_{2, q} \leqslant \frac{C}{q-1}\|g\|_{q} \text { for } 1<q \leqslant 2 .
$$

In fact, if $\Omega$ has a smooth boundary (for instance $C^{1,1}$ ), (8) can be shown by the classical proof [3], examining carefully the constants involved. In the case of a Lipschitz convex domain this result was proven recently by $\mathrm{T}$. Wolff in unpublished work. Indeed, he has proven a weak type inequality for $L^{1}$ that, together with the known result for $q=2$, implies (8) by usual interpolation methods.

By the known duality argument of Aubin-Nitsche [1], and using (8), we get

$$
\left|u-u_{h}\right|_{0, p} \leqslant C p h^{2}\|u\|_{2, p}, \quad 2 \leqslant p<\infty,
$$

with $C$ independent of $p$.

But, in general, if we have two functions $g_{1}$ and $g_{2}$ such that

$$
\left|g_{1}\right|_{0, p} \leqslant C_{1} p\left|g_{2}\right|_{0, p}, \quad 2 \leqslant p<\infty,
$$

then,

$$
\left|g_{1}\right|_{0, \phi_{1}} \leqslant C_{1} C_{2}\left|g_{2}\right|_{0, \infty}
$$

where $C_{2}$ depends only on $\Omega$. In fact,

$$
\begin{aligned}
\int_{\Omega} \phi_{1}\left(\frac{\left|g_{1}(x)\right|}{K\left|g_{2}\right|_{0, \infty}}\right) d x & =\int_{\Omega} \sum_{j=2}^{\infty} \frac{\left|g_{1}(x)\right|^{j}}{K^{j}\left|g_{2}\right|_{0, \infty}^{j}} \frac{1}{j !} d x \\
& =\sum_{j=2}^{\infty} \frac{1}{j ! K^{j}\left|g_{2}\right|_{0, \infty}^{j}} \int_{\Omega}\left|g_{1}(x)\right|^{j} d x \leqslant \sum_{j=2}^{\infty} \frac{C_{1}^{j} j^{j}\left|g_{2}\right|_{0, j}^{j}}{j ! K^{j}\left|g_{2}\right|_{0, \infty}^{j}} \\
& \leqslant \sum_{j=2}^{\infty}\left(\frac{C_{1}}{K}\right)^{j} \frac{j^{j}}{j !}|\Omega|,
\end{aligned}
$$

and the last series is convergent and less than 1 if we choose $K=C_{1} C_{2}$ with $C_{2}$ sufficiently large, depending only on $\Omega$.

Department of Mathematics

University of Chicago

Chicago, Illinois 60637

1. P. G. Ciarlet, The Finite Element Method for Elliptic Problems, North-Holland, Amsterdam, 1978.

2. T. K. Donaldson \& N. S. Trudinger, “Orlicz-Sobolev spaces and imbedding theorems," J. Funct. Anal., v. 8, 1971, pp. 52-75. 
3. D. Gilbarg \& N. S. Trudinger, Elliptic Partial Differential Equations of Second Order, SpringerVerlag, Berlin and New York, 1983.

4. R. Haverkamp, “Eine Aussage zur $L^{\infty}$-Stabilität und zur genauen Konvergenzordnung der $H_{0}^{1}$-Projektionen," preprint 508, Sonderforschungsbereich 72, Approximation und Optimierung, Universität Bonn, 1983.

5. F. John \& L. Nirenberg, "On functions of bounded mean oscillation," Comm. Pure Appl. Math., v. 14, 1961, pp. $415-426$.

6. J. PeEtre, “On convolution operators leaving $L^{p . \lambda}$ invariant,” Ann. Mat. Pura Appl., v. 72, 1966, pp. 295-304.

7. R. Rannacher, "Zur $L^{\infty}$-Konvergenz linearer finiter Elemente beim Dirichlet-Problem," Math. $Z$., v. 149,1976 , pp. 69-77.

8. R. Rannacher \& R. Scott, "Some optimal error estimates for piecewise linear finite element approximations," Math. Comp., v. 38, 1982, pp. 437-445.

9. A. H. Schatz \& L. B. WAhlbin, "On the quasi-optimality in $L_{\infty}$ of the $H^{1}$-projection into finite element spaces," Math. Comp., v. 38, 1982, pp. 1-22. 